\title{
Theoretical Determination of the Optimal Fiber Volume Fraction and Fiber-Matrix Property Compatibility of Short Fiber Composites*
}

\author{
NING PAN \\ Division of Textiles and Clothing \\ University of California \\ Davis, California 95616
}

\begin{abstract}
Although the question of minimum or critical fiber volume fraction, beyond which a composite can then be strengthened due to addition of fibers, has already been dealt with by several investigators for both continuous and short fiber composites, a study of maximum or optimal fiber volume fraction at which the composite reaches its highest strength has not been reported. The present analysis has investigated this issue for short fiber case based on the well-known shear lag (the elastic stress transfer) theory. Using the relationships obtained, the minimum spacing between fibers is determined upon which the maximum fiber volume fraction can be calculated. The effects on the value of this maximum fiber volume fraction due to such factors as the mechanical properties of the fiber and matrix, the fiber aspect ratio, and fiber packing forms are discussed. Furthermore, combined with the previous analysis on the minimum fiber volume fraction, this maximum fiber volume fraction is used to examine the property compatibility of fiber and matrix in forming a composite. This is deemed to be useful for composite design. Some examples are provided as well to illustrate the results.
\end{abstract}

\section{INTRODUCTION}

$I^{t}$ $t$ has been well known that if very few fibers are added to a matrix, the material is weakened rather than strengthened. Therefore there has to be a minimum critical fiber volume fraction $V_{\min }$, above which the fiber reinforcing function can be realized. Studies addressing this problem derived specific values of $V_{\min }$ for various cases and under different conditions $(1,8,11)$. On the other hand, however, as the fiber content in the matrix is being increased, the tensile strength of the composite will increase to a certain point where, upon further increasing of fiber amount, the bonding between the fibers and the matrix will start to deteriorate as the fibers become too close to each other. As a result, for a short-fiber composite, the tensile strength will decline due to the bond failure of the system caused by excessive fibers. Therefore there will be a maximum value of fiber volume fraction as well, that being the upper limit of fiber amount allowable in the system for reinforcement.

There have been no reported studies on this maximum value of fiber volume fraction, as far as the present author is aware. This may be due mainly to the fact that, in most cases the fiber amount which

\footnotetext{
* Presented at the $\mathrm{V}$. Conference on Advanced Engineering Fibers and Textile Structures for Composite, Raleigh. N.C., October 1991.
}

can be incorporated into a matrix system is limited by the processing technology (4). It may be difficult for fiber volume fraction to reach its theoretical maximum allowable value. Therefore, the maximum fiber volume issue may not be as significant as the minimum one for practical applications. Nevertheless, study on this subject is still desirable partly due to its theoretical significance. More importantly, as shown in this article, the investigation of this issue can not only provide the maximum value of fiber volume fraction, but also determine the property compatibility of various fiber and matrix materials for composite design so as to achieve optimum composite strength and full material usage.

The present study deals with this problem based on a shear strength criterion within the composite. The effects of fiber length and fiber orientation are also investigated.

\section{THE MINIMUM ALLOWABLE SPACING BETWEEN FIBERS IN A SHORT FIBER COMPOSITE}

It has been a well known mechanism that when a fiber composite is under a uniaxial tension, the axial displacements in the fiber and in the matrix will be different because of the differences in tensile properties of these two components. As a result, shear strains will be created on all planes parallel to the 
axes of the fibers. The shear strain and the resulting shear stress are the primary means by which load is transferred to fibers (for a short fiber composite), or distributed between and supported by the two components of composites. It is through this interaction between fibers and matrix that fiber reinforcing function is realized. There have been several theories trying to explain this fiber-matrix interaction. The first one was entirely based on elastic mechanism proposed by Cox (3) in 1952, and is now referred to as the shear lag theory, and another similar version was later proposed by Rosen (12). Since then, a number of new theories were suggested such as the slip theory (7) to account for matrix plasticity at the fiber surface near the fiber ends, applicable to well-bonded reinforced metals, and the theory of frictional sliding (7) to reinforced polymers and ceramics. In the present study, the model of the elastic stress transfer is used as the main theoretical basis. As it will be shown, although this theory explains the behavior of composites at low stress, it provides adequate information to determine the maximum fiber volume fraction for design purposes. Furthermore the analysis will be helpful in the later attempt to look into the case of inelastic interaction.

Assumptions made in this analysis include:

1. Since the elastic model is used here, conclusions from the present analysis are valid only if the original assumptions associated with this model hold.

2. The composite consists of identical short fibers, each with length $l$, circular cross-section area $A_{f}$ of uniform radius $r$, and tensile modulus $E_{f}$.

3. All fibers are distributed uniformly along the length of the composite so that the fiber area fractions on all the cross sections of the composite are constant.

4. Both fibers and matrix behave elastically, and the interface transfers the stress between fibers and matrix without yielding or slipping.

5. Fiber ends are all normal so that the shape effect of fiber end on the stress transfer (6) is excluded in this analysis.

6. Furthermore, the fiber-fiber interaction within the composite and the effect of matrix property change as a result of the fiber interfering with dislocation motion in the matrix are also ignored.

Take the mean fiber center-to-center spacing normal to their length to be $2 R$ (see Fig. 1). Assume the composite as a whole is subject to a strain $\epsilon_{c}$ which will cause a strain $\epsilon_{f}$ in a fiber. If $P$ is the load in the fiber at a distance $x$ from the fiber end, then according to Cox (3), the distribution of tensile stress in this arbitrary fiber is

$$
\sigma=\frac{P}{A_{f}}=E_{f} \epsilon_{f}\left[1-\frac{\cosh \beta\left(\frac{l}{2}-x\right)}{\cosh \beta \frac{l}{2}}\right]
$$
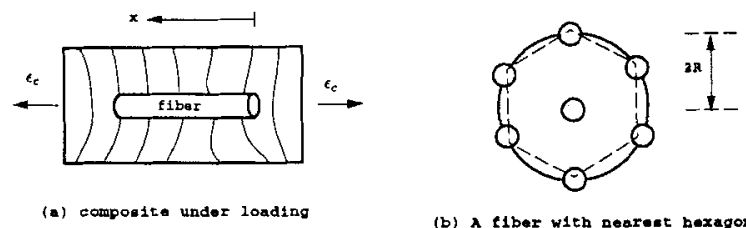

(a) Compoeite under loading

(b) A tiber with nearest hexagonaliy packed nenghbor:
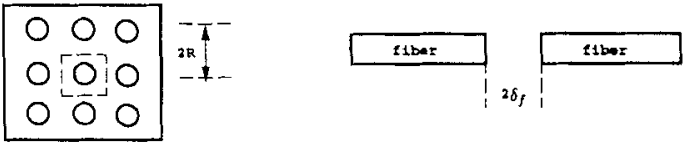

(c) A fiber with nearest scuare-packed ne1ghbore

Fig. 1. Fiber embedded in a matrix and fiber packing forms.

where

$$
\beta=\frac{1}{r} \sqrt{\frac{G_{m}}{E_{f}}\left(\frac{2}{\ln (R / r)}\right)}
$$

and $G_{m}$ is the shear modulus of the matrix. Note that $\sigma=0$ at $x=0$, and $l$.

The maximum stress occurs at the middle of position $x=l / 2$ as

$$
\sigma_{\max }=E_{f} \epsilon_{f}\left[1-\frac{1}{\cosh \beta \frac{l}{2}}\right]
$$

It can be seen from $E q 3$ that for given fiber strain, and fiber and matrix properties, the fiber-fiber spacing $R$ is the key factor in determining the magnitude of the tensile stress.

If $\tau$ is the shear stress in the direction of the fiber axis, on planes parallel to this axis, then at the fiber surface we have also due to $\operatorname{Cox}(3)$

$$
\tau=E_{f} \epsilon_{f} \sqrt{\frac{G_{m}}{2 E_{f} \ln (R / r)}} \frac{\sinh \beta\left(\frac{l}{2}-x\right)}{\cosh \beta \frac{l}{2}}
$$

The maximum value of $\tau$ occurs at the fiber ends, i.e. at $x=0$ and $l$

$$
\tau_{\max }=E_{f} \epsilon_{f} \sqrt{\frac{G_{m}}{2 E_{f} \ln (R / r)}} \tanh \beta \frac{l}{2}
$$

and it is zero at the middle of the fiber.

The ratio of the maximum value of shear stress to the maximum tensile stress in the fiber is

$$
\frac{\tau_{\max }}{\sigma_{\max }}=\sqrt{\frac{G_{m}}{2 E_{f} \ln (R / r)}} \operatorname{coth} \beta \frac{l}{4}
$$

This ratio is of great importance as it represents the fiber and matrix properties as well as the fiber spacing $R$ within the matrix, and is independent of composite strain. It is therefore a property indicator of the 
composite. The validity of Eqs $1-6$ has been verified by several experimental studies $(6,7)$.

In reinforcing the composite to its maximum tensile strength, the tensile strength of fibers has to be fully utilized. In other words, a stress equal to the tensile breaking stress of the fibers $\sigma_{b f}$ must be reached at the middle of the fibers, i.e., $\sigma_{\max }=\sigma_{b f}$. So Eq 6 can be rearranged into

$$
\tau_{\max }=\sigma_{b f} \sqrt{\frac{\bar{G}_{m}}{2 E_{f} \ln (R / r)}} \operatorname{coth} \beta \frac{l}{4}
$$

This equation gives the relationship between fiber spacing $R$ (or the spacing ratio $R / r$ ) and the maximum value of shear stress. When $R$ decreases, the value of $\tau_{\max }$ will increase as shown in Fig. 2 . The minimum spacing $R_{\min }$ is thus determined when $\tau_{\max }$ has been increased to the shear strength of the matrix adjacent to the interface or the shear strength of the fiber/matrix interface, whichever is less, designated as $\tau_{s}$. Because of the elastic assumption where the matrix can not deform plastically, this will cause either the fiber/matrix interface or the matrix to fail in shear.

Furthermore, replacing $\tau_{\max }$ by $\tau_{s}$ and rearranging $E q 7$ gives the following expression which governs the relationship between the minimum spacing ratio $R_{\text {min }} / r$ and the strength ratio $\sigma_{b f} / \tau_{s}$, the fiber aspect ratio $l / r$ as well as the modulus ratio $G_{m} / E_{f}$

$$
\begin{aligned}
& \ln \left(R_{\min } / r\right) \\
& \quad=\left(\frac{\sigma_{b f}}{\tau_{s}} \operatorname{coth} \beta \frac{l}{4}\right)^{2} \frac{G_{m}}{2 E_{f}} \\
& =\frac{G_{m}}{2 E_{f}}\left(\frac{\sigma_{b f}}{\tau_{s}}\right)^{2}\left(\operatorname{coth}\left[\frac{1}{4} \frac{l}{r} \sqrt{\frac{G_{m}}{E_{f}} \frac{2}{\ln \left(R_{m i n} / r\right)}}\right]\right)^{2}
\end{aligned}
$$

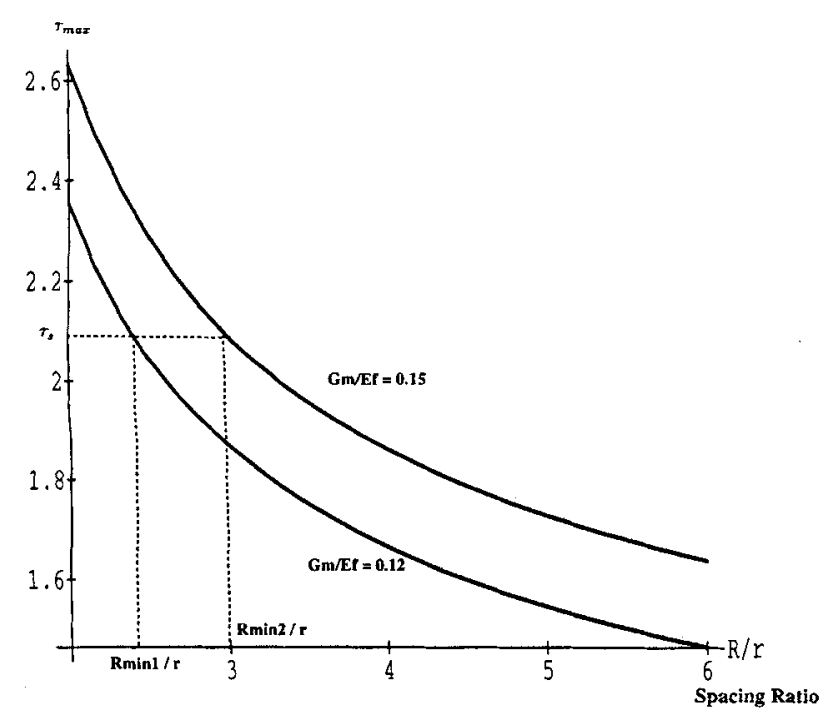

Fig. 2. Maximum shear stress us. spacing ratio.
This is a transcendental equation for $R_{\min } / r$, and its solution can only be calculated numerically.

However if the fiber length is relatively long so that $\operatorname{coth} \beta l / 4 \rightarrow 1$, we then have an explicit relationship between the fiber spacing ratio and the fiber-matrix properties

$$
\ln \left(R_{\min } / r\right)=\left(\frac{\sigma_{b f}}{\tau_{s}}\right)^{2} \frac{G_{m}}{2 E_{f}}
$$

or

$$
R_{\text {min }} / r=e\left(\frac{\sigma_{b f}}{\tau_{s}}\right)^{2} \frac{G_{m}}{2 E_{f}}
$$

In this analysis, the effect of stress transfer across the fiber ends, which will cause an extra load on both fiber and matrix in this region, is neglected, because this effect has been considered insignificant (11) as long as the fiber aspect ratio $l / r>10$. Also the influence of stress concentration across the fiber ends, which will lead to a greater shear stress (8) and will affect the slippage behavior of the fiber ends, is ignored.

In addition, in the present analysis, fiber and the matrix are assumed as completely elastic. This is an ideal case and only valid in practice to brittle materials. For some cases where plastic deformation of the matrix does exist, the conclusion drawn from this study will be a conservative one and certain modification may be needed, since the plastic deformation of the matrix will alleviate the shear stress. A different model of the spacing/stress relationship will be needed, however, for a matrix that is significantly plastic and flows under loading, or for structures where the effect of frictional sliding between fiber and matrix during the stress transfer is not negligible.

\section{THE MAXIMUM FIBER VOLUME FRACTION IN COMPOSITES}

As indicated above, since there is a minimum $R_{\text {min }} / r$ value between fibers within a composite below which the structure will collapse due to shear failure, this minimum spacing will correspondingly define an upper limit of fiber amount which is allowable to be incorporated into a given matrix. Further, the composite will reach its highest strength at this maximum fiber volume fraction $V_{\text {max }}$, as there is maximum amount of fibers in the composite and each is fully utilized. In other words, this maximum fiber volume fraction is also the optimal value in maximizing the composite tensile strength. Obviously the specific value of $V_{\max }$ is dependent on, besides the parameters included in the equation, the forms of fiber arrangement within a composite as well. In addition, for the short fiber case where fiber ends don't meet, the maximum fiber volume fraction also varies with the distance between fiber ends. Let us assume this distance between the ends of two fibers in a composite to be $2 \delta_{f}$ as shown in Fig. Id.

The following are the two cases of fiber arrangement most often encountered in a composite (11). 


\section{Hexagonally Packed Fibers}

The fiber arrangement of this type is schematically shown in Fig. 1 b. Suppose there are totally $N$ fibers within the composite. Considering the hexagonal element with the area enclosed by the dotted line in Fig. $1 b$, and according to the definition of fiber volume fraction of a composite, we have the maximum fiber volume fraction in this case

$$
\begin{aligned}
V_{f m h} & =\frac{V_{\text {fiber }}}{V_{\text {total }}}=\frac{3 \pi r^{2} l}{\frac{3 \sqrt{3}}{2}\left(2 R_{\text {mir }}\right)^{2}\left(l+2 \delta_{f}\right)} \\
& =\frac{\pi}{2 \sqrt{3}}\left(\frac{r}{R_{\min }}\right)^{2}\left(\frac{1}{1+2 \delta_{f} / l}\right)
\end{aligned}
$$

For the case when the fiber length is so long as $l \gg \delta_{f}$ that the fiber end effect can be neglected, the expression becomes

$$
V_{f m h}=\frac{\pi}{2 \sqrt{3}}\left(\frac{r}{R_{\min }}\right)^{2}
$$

Because of this direct relationship between the maximum fiber volume fraction and the minimum fiber spacing ratio, it will be considered equivalent in the later analysis to refer to either of them.

If all fibers were packed so closely that

$$
R_{\min }=r, \quad \delta_{f}=0
$$

the maximum fiber volume fraction would be

$$
V_{f m h}=\frac{\pi}{2 \sqrt{3}}
$$

\section{Square-Packed Fibers}

The fiber arrangement in this case is shown in Fig. lc and we have accordingly

$$
V_{f m s}=\frac{\pi r^{2} l}{\left(2 R_{\min }\right)^{2}\left(l+2 \delta_{f}\right)}=\frac{\pi}{4}\left(\frac{r}{R_{\min }}\right)^{2}\left(\frac{1}{1+2 \delta_{f} / l}\right)
$$

For long fiber case, it becomes

$$
V_{f m s}=\frac{\pi}{4}\left(\frac{r}{R_{\min }}\right)^{2}
$$

In the closely packed case, there would be

$$
V_{f m s}=\frac{\pi}{4}
$$

It can be seen by comparing $E q 11$ and 15 that in either of the two packing forms, the value of maximum fiber volume fraction monotonically increases as the fiber spacing decreases. The relationship of or the difference between the maximum fiber volume fractions of these two packing forms is given by

$$
\frac{V_{f m s}}{V_{f m h}}=\frac{\sqrt{3}}{2}
$$

That is, the maximum possible fiber volume fraction for square-packed fibers is less than that of hexagonally packed case. Because of this relationship between the two fiber packing forms, for briefness, only the square-packed form is used in the following analysis.

Note that when the effect of fiber orientation is considered, the fiber arrangement may not be as regular as the two examples shown here. Consequently the value of the actual maximum fiber volume fraction may be lower than the present results.

\section{THE MINIMUM FIBER VOLUME FRACTION IN COMPOSITES}

In the following analysis, $\epsilon_{b f}, \epsilon_{b m}$, and $\epsilon_{y m}$ represent the fiber breaking strain, the matrix breaking strain, and the matrix yield strain.

In a composite, there are possible three cases

1. $\epsilon_{b f}<\epsilon_{y m}$,

2. $\epsilon_{y m}<\epsilon_{b f}<\epsilon_{b m}$

3. $\epsilon_{b m}<\epsilon_{b j}$.

and each of them will lead to different failure behavior of the composite. Usually, however, the breaking strain of the fiber $\epsilon_{b f}$ is less than the yield strain of the matrix $\epsilon_{y m}$ so that only the first case is considered here. The treatments of two other cases, however, are in principle the same.

If the variations of fiber tensile properties are ignored, it is well known that the breaking strength of the composite $\sigma_{b c}$ is of the contributions from both fibers and the matrix, and can be expressed as in (1)

$$
\sigma_{b c}=\sigma_{f m}\left(1-V_{f}\right)+\eta_{l} \eta_{\theta} \sigma_{b f} V_{f}, \quad V_{f}>V_{\min }
$$

where $\sigma_{b f}$ is the breaking strength of the fiber, and $\sigma_{f m}$ is the stress on the matrix at the breaking tensile: strain of the fiber. The factors $\eta_{l}$ and $\eta_{t}$ account for the effects of limited fiber length for the short fiber case, and of fiber orientation, and are often called the length efficiency and fiber orientation efficiency factors. $V_{\min }$ is the minimum value of the fiber volume fraction which must be exceeded if the strength of the composite is to be given by the Law of Mixture as shown above. The value of $V_{\min }$ has been determined according to, for example, Kelly (8) as

$$
V_{\min }=\frac{\sigma_{b m}-\sigma_{f m}}{\eta_{l} \eta_{t} \sigma_{b f}+\sigma_{b m}-\sigma_{f m}}
$$

where $\sigma_{b m}$ is the breaking strength of the matrix.

For a continuous fiber composite where all fibers are aligned in the loading direction, there will be

$$
\eta_{l}=1, \quad \eta_{\theta}=1
$$

and we then have

$$
V_{\min }=\frac{\sigma_{b m}-\sigma_{f m}}{\sigma_{b f}+\sigma_{b i n}-\sigma_{f m}}
$$




\section{DETERMINATION OF THE FIBER LENGTH EFFICIENCY FACTOR}

It has been claimed (9) that in the post-cracking stage the combined effect due to short fiber length and fiber orientation cannot be simply calculated as the product of the length efficiency factor and the orientation efficiency factor because the orientation efficiency factor is also a function of the fiber length. For the elastic model of pre-cracking stage as in the present case, these two factors can be considered independent of each other and can hence be determined separately.

The length efficiency factor, specifying the effect of a definite length of fibers in short fiber composite, has two expressions depending on the stress interaction mechanisms (9). For the inelastic case, the most common version of this fiber length efficiency factor is expressed in terms of critical fiber length $(1,8,9)$. For elastic case, however, it can be easily determined based on the tensile stress distribution. From $E q 1$, the average tensile stress over the length of this short fiber can be calculated as

$$
\bar{\sigma}_{f l}=E_{f} \epsilon_{f}\left[1-\frac{\tanh \beta \frac{l}{2}}{\beta \frac{l}{2}}\right]
$$

When fiber length $l \rightarrow \infty$, it becomes

$$
\bar{\sigma}_{f l}=\sigma_{f l}=E_{f} \epsilon_{f}
$$

Therefore the fiber length efficiency factor is defined as

$$
\eta_{l}=1-\frac{\tanh \beta \frac{l}{2}}{\beta \frac{l}{2}}
$$

This expression shows that, compared to the continuous fibers, the tensile stress on a short fiber is discounted by a factor $\eta_{l}$ due to limited fiber length.

It is easy to prove that when $l \rightarrow 0, \eta_{l}=0$.

\section{DETERMINATION OF FIBER ORIENTATION EFFICIENCY FACTOR}

In most published studies where the effect of fiber orientation was considered, fibers were assumed either all aligned in the same direction at a fixed angle with respect to the axis of the composite loading direction $(1,8,11)$, i.e., there is no variation or spread existing in fiber orientation, or fibers distributed totally in random $(3,9)$. Yet there are some other cases as well.

Although it is usually desirable to orient the fibers in the loading direction to enhance stiffness and strength properties, in short-fiber composites, it is normally very difficult, if not impossible, to achieve perfect alignment or completely random distribution of short fibers. The orientation distributions of fibers in a composite are determined by the processing con- ditions. Partial fiber alignment is typical in injection and transfer molded composites, while planar partial random orientation is typical in sheet molding compounds (2). Therefore for most cases, variation of fiber orientation is unavoidable and has to be included in the study. There have been several reports $(2,5,13,14)$ dealing with fiber orientation spreading. A more explicit form of the expression of this fiber orientation efficiency factor is obtained in the present study.

\section{Form of Fiber Orientation Density Function}

Since it is impractical to deal with fibers of different orientations individually, a statistical approach is usually a better alternative. To do this, a known form of the function to describe the fiber orientation probability density is the premise.

Two cases of fiber orientation distribution are of practical importance (2). In the case of injection molded objects, fiber orientation distribution is independent of the base angle if the direction of flow is along the composite principal axis. In sheet molding compounds it is reasonable to assume that the short fibers all lie within a plane and the problem is reduced to a two-dimensional one. In either case, by properly arranging the coordinate system, the fiber orientation density function can be expressed as

$$
\Omega(\theta)= \begin{cases}\frac{1}{\alpha} & 0 \leq \theta \leq \alpha \\ 0 & \alpha \leq \theta<\pi / 2\end{cases}
$$

where $\theta$ is the polar angle of a fiber with respect to the composite principal axis, and $\alpha$ is the limit of $\theta$.

\section{Relationship Between Strains of Composite, Matrix and Fiber}

Assume the composite as a whole is subject to a strain $\epsilon_{c}$ which will cause strain $\epsilon_{f}$ in the fiber and $\epsilon_{m}$ in the matrix. It has been widely accepted that as the elastic stress transfer mechanism is dominant at the pre-cracking stage, the longitudinal displacements of the fiber and matrix interface are considered geometrically compatible. In other words, the matrix strain will be the same as the composite strain before cracking. The fiber strain however is dependent on the fiber orientation with respect to the loading direction.

There are several approaches in finding the relationship between the composite strain and fiber strain, such as the tensor transformation method (10) and the affine deformation model (5). However, as shown below, a few simple differentiation operations can also derive the same result.

Let us consider a cylinder of matrix material with height $H$ and radius $R_{c}$. Inside the matrix there is a fiber with length $l$ and orientation $\theta$ (see Fig. 3). There is a relationship between the three variables

$$
l^{2}=R_{c}^{2}+H^{2}
$$




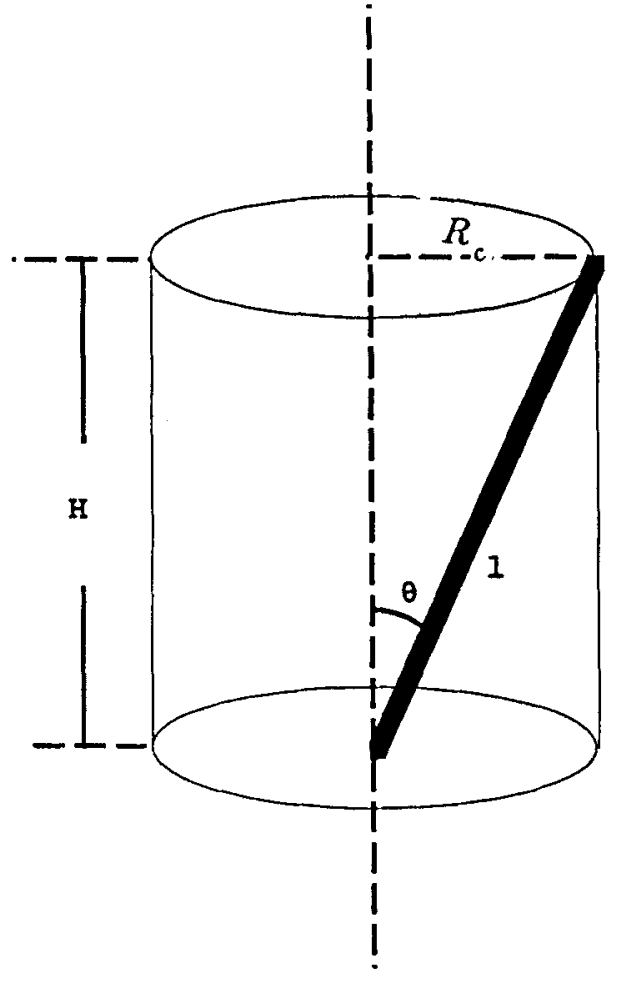

Fig. 3. Geometrical relationships between fiber and matrix.

Differentiating the both sides gives

$$
2 l d l=2 R_{c} d R_{c}+2 H d H
$$

It can be further expressed as

$$
\frac{d l}{l}=\frac{R_{c}^{2}}{l^{2}} \frac{d R_{c}}{R_{c}}+\frac{H^{2}}{l^{2}} \frac{d H}{H}
$$

Bringing

$$
\frac{d H}{H}=\epsilon_{c}
$$

and

$$
\frac{d R_{c}}{R_{c}}=-\nu_{m} \epsilon_{c}
$$

into it yields

$$
\epsilon_{f}=\frac{d l}{l}=\epsilon_{c}\left(\cos ^{2} \theta-\nu_{m} \sin ^{2} \theta\right)
$$

where $\nu_{m}$ represents the matrix Poisson's ratio. Note that, similar to previous analyses $(5,8)$, the effect of the fiber Poisson's ratio has been excluded in the analysis. Equation 30 has been found to be consistent with both the experimental data and the results based on other more sophisticated analytical analysis in (5). However, since the change of the fiber orientation during composite deformation is neglected in the above analysis, it is preferable to apply the conclusion to small strain case.

\section{The Result of Fiber Orientation Efficiency Factor}

Once we have the relationship between fiber strain and the overall composite strain, the average strain on an arbitrary fiber due to its orientation can then be calculated as

$$
\bar{\epsilon}_{f}=\int_{0}^{\alpha} \epsilon_{c}\left(\cos ^{2} \theta-\nu_{m} \sin ^{2} \theta\right) \Omega(\theta) d \theta
$$

Bringing the distribution function into the above equation gives

$$
\bar{\epsilon}_{f}=\frac{\epsilon_{c}}{4 \alpha}\left[2 \alpha\left(1-\nu_{m}\right)+\left(1+\nu_{m}\right) \sin 2 \alpha\right]
$$

The overall average tensile stress on this fiber thus becomes

$$
\bar{\sigma}_{f}=\eta_{l} E_{f} \bar{\epsilon}_{f}=\eta_{l} E_{f} \frac{\epsilon_{c}}{4 \alpha}\left[2 \alpha\left(1-\nu_{m}\right)+\left(1+\nu_{m}\right) \sin 2 \alpha\right]
$$

Furthermore, because of fiber misorientation, the contribution of this fiber toward the composite strength will be discounted according to the equation in (9)

$$
\left[\sigma_{f c}\right]=[T]\left[\sigma_{f}\right]
$$

where $[T]$ is the transformation matrix, and $\left[\sigma_{f}\right]$ and $\left[\sigma_{f c}\right]$ are the actual fiber stress tensor and the fiber stress tensor in the orthogonal directions with respect to loading direction. For the present uniaxially loading case where the composite principal axis is the loading direction, the above equation reduces to

$$
\sigma_{f c}=\bar{\sigma}_{f} \cos ^{2} \theta-2 \bar{\tau}_{f} \sin \theta \cos \theta
$$

It can be easily proven from $E q 4$ that

$$
\bar{\tau}_{f}=0
$$

So we have

$$
\sigma_{f c}=\bar{\sigma}_{f} \cos ^{2} \theta
$$

Because of the variation of fiber orientation, the average value of the stress over all fibers is

$$
\begin{aligned}
\bar{\sigma}_{f c}= & \int_{0}^{\alpha} \bar{\sigma}_{f} \cos ^{2} \theta \Omega(\theta) d \theta \\
= & \eta_{l} E_{f} \epsilon_{c} \frac{1}{16 \alpha^{2}}\left[2 \alpha\left(1-\nu_{m}\right)\right. \\
& \left.\quad+\left(1+\nu_{m}\right) \sin 2 \alpha\right](2 \alpha+\sin 2 \alpha)
\end{aligned}
$$

The fiber orientation efficiency factor is thus derived as

$\eta_{\theta}=\frac{1}{16 \alpha^{2}}\left[2 \alpha\left(1-\nu_{m}\right)+\left(1+\nu_{m}\right) \sin 2 \alpha\right](2 \alpha+\sin 2 \alpha)$

It can be proved that when $\alpha \rightarrow 0, \eta_{\theta}=1$. The minimum value of $\eta_{\theta}=\left(1-\nu_{m}\right) / 4$ is achieved when $\alpha \rightarrow$ $\pi / 2$. 


\section{THE PROPERTY COMPATIBILITY OF FIBER AND MATRIX IN COMPOSITES}

Now that we have determined the maximum (allowable) fiber volume fraction and the minimum (necessary) fiber volume fraction, we can use these two values to examine the fiber-matrix property compatibility.

Obviously, for composite design with any possible combinations of fiber and matrix, the criterion

$$
V_{\max } \geq V_{\min }
$$

has to be satisfied. Since these two values are determined by the properties of the fiber and matrix as well as the spacing between fibers, Eq 40 actually provides the restraints on the permissible values of all these parameters in a composite. So this equation can be used to study the fiber-matrix property compatibility in materials selection for a composite.

The easier way of using this equation is to study the boundaries enclosed by the curves of these two volume fractions corresponding to different parameters. Some specific examples are shown in the next section.

\section{CALCULATION AND DISCUSSION}

First of all, as we have had all the equations describing the relationships between the composite structural parameters and the constituent materials properties, a parametric study becomes possible to show the effects of these variables. The data used for calculation are listed in Table 1. For generality, the ratios of fiber and matrix properties are used wherever possible. When the effect of a specific parameter in Table 1 is investigated over the given range, other parameters will take the typical values provided. The results are illustrated in Figs. 4 to 10 .

Figure 4 shows the effect of the Strength Ratio $\sigma_{b f} / \tau_{s}$ on the values of $V_{\max }$ at long and short fiber lengths. As the strength ratio increases, meaning

Table 1. The Fiber Matrix Properties Used for Calculation

\begin{tabular}{lll}
\hline \multicolumn{1}{c}{ Item } & \multicolumn{1}{c}{ Range } & Typical \\
\hline Strength ratio $\frac{\sigma_{b f}}{\tau_{s}}$ & $1.5-5.0(7)$ & 2.4 \\
Modulus ratio $\frac{G_{m}}{E_{f}}$ & $0.02-0.3(7)$ & 0.03 \\
Fiber aspect ratio $\frac{1}{r}$ & $5-200$ & 60 \\
Fiber breaking strength $\sigma_{b f}$ & $4-20 \mathrm{Gpa}(9)$ & $8 \mathrm{Gpa}$ \\
Matrix stress difference & $0.2-4.0 \mathrm{Gpa}$ & $2 \mathrm{Gpa}$ \\
$\sigma_{b m}-\sigma_{t m}$ & $($ assumed) & $\pi$ \\
Fiber orientation range $\alpha$ & $0-\frac{\pi}{3}$ (assumed) & $\frac{\pi}{6}$ \\
Matrix Poisson's ratio $\nu_{m}$ & $0.2-0.5$ (assumed) & 0.3 \\
Fiber end spacing & $0.0008-0.05$ & 0.003 \\
length ratio $\frac{\delta_{f}}{l}$ & (assumed) & \\
\hline
\end{tabular}

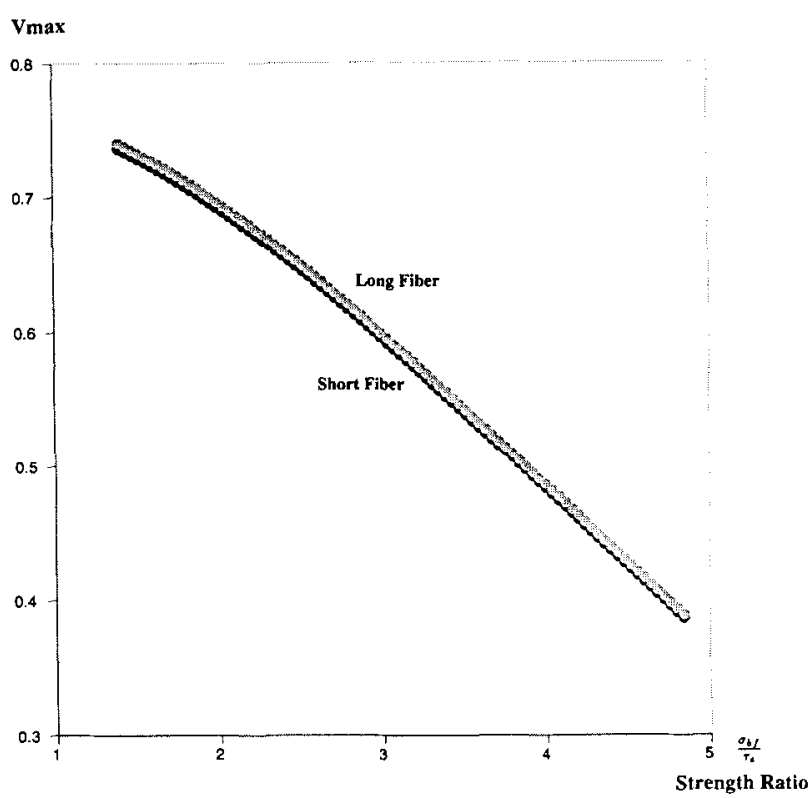

Fig. 4. Maximum volume fraction vs. strength ratio.

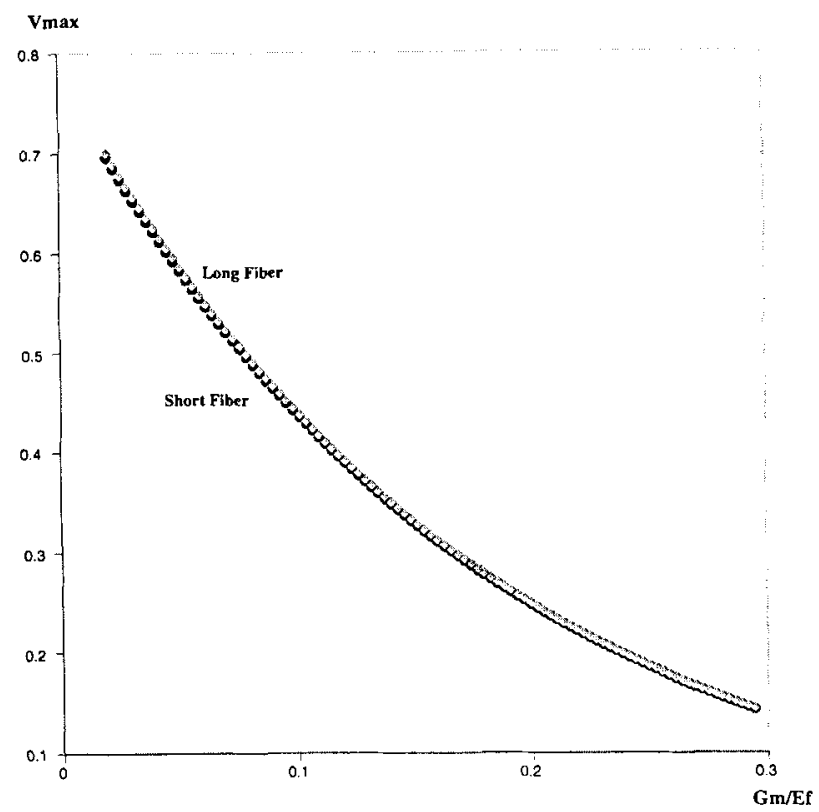

Fig. 5. Maximum volume fraction us. modulus ratio.

stronger fibers are used, or a weaker bonding shear strength exists between the matrix and fibers, $V_{\max }$ will decrease, indicating a greater spacing between fibers is required in order to maintain a stable structure. Note that fiber length does not have significant effect on the result.

The effect of the modulus ratio $G_{m} / E_{f}$ on $V_{\max }$ value is illustrated in Fig. 5. It is also a monotonically decreasing relationship. This means that a matrix with higher shear modulus or a less stiff fiber will result in a smaller $V_{\max }$ value, or require greater spacing between fibers. Consequently the tensile 


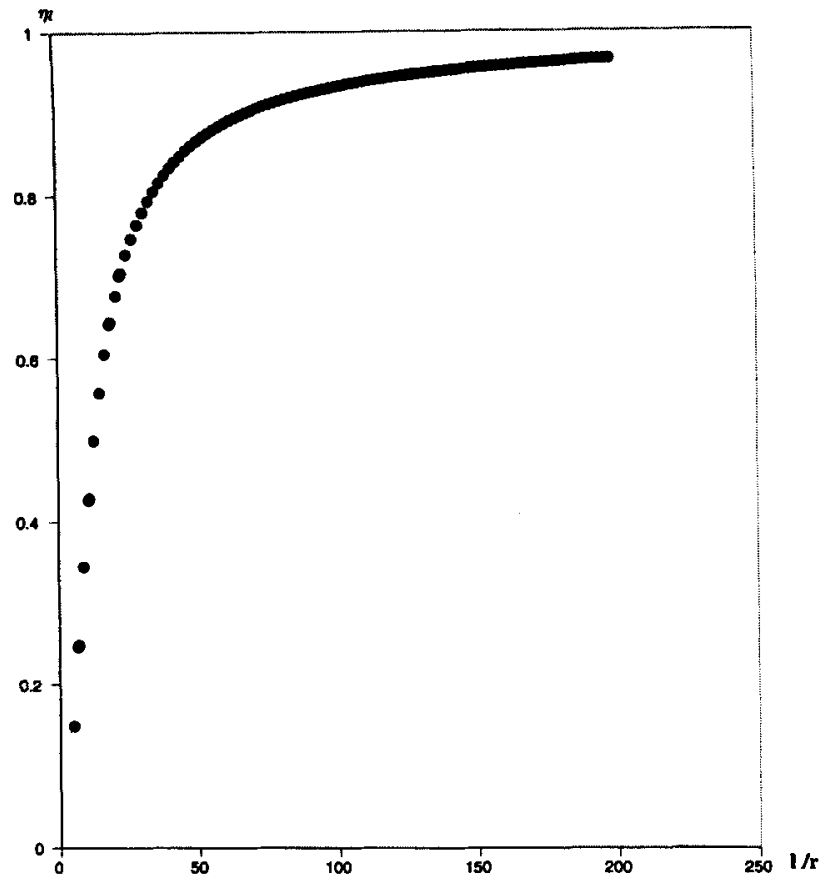

Fig. 6. Length efficiency factor vs, fiber aspect ratio.

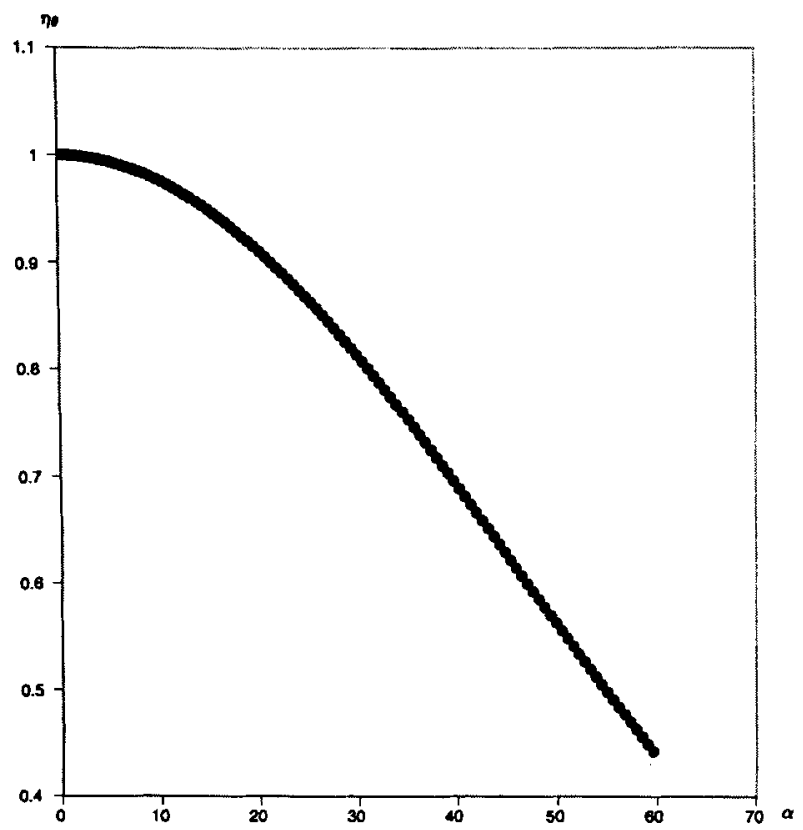

Fig. 7. Relationship between orientation range and efficiency factor.

strength of the composite will be lower. Again there is no noticeable difference caused by fiber length.

Figures 6 and 7 show the relationships between the fiber length efficiency factor $\eta_{l}$ and the fiber aspect ratio $l / r$, and between the fiber orientation range $\alpha$ and the fiber orientation efficiency factor $\eta_{\theta}$ respectively. As shown in the results, increasing of fiber aspect ratio (a longer or thinner fiber) will raise the fiber length efficiency factor, and a wider spread (a

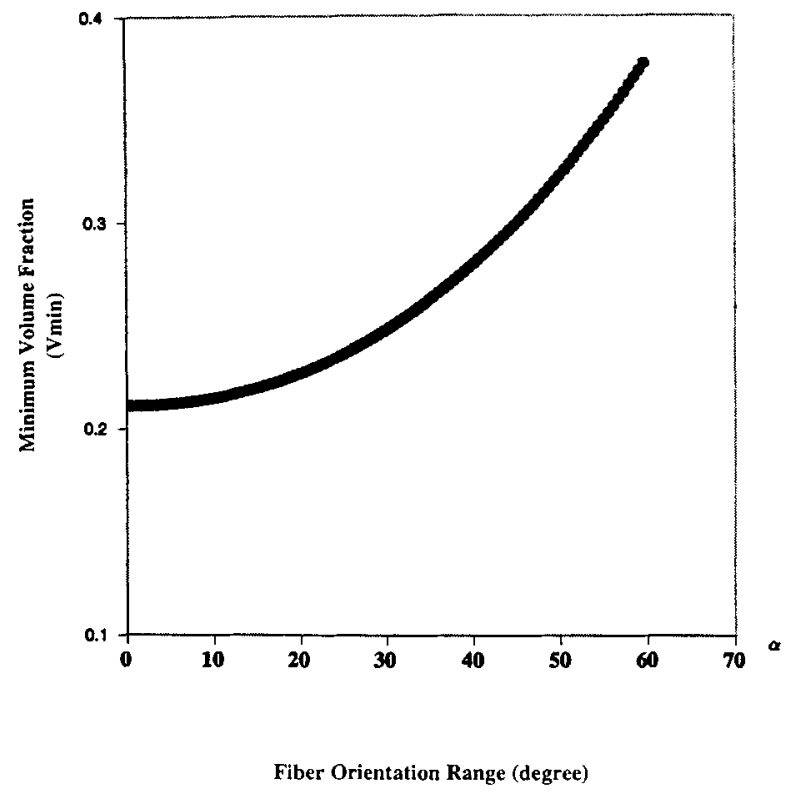

Fig. 8. Fiber orientation range vs. fiber minimum volume fraction.

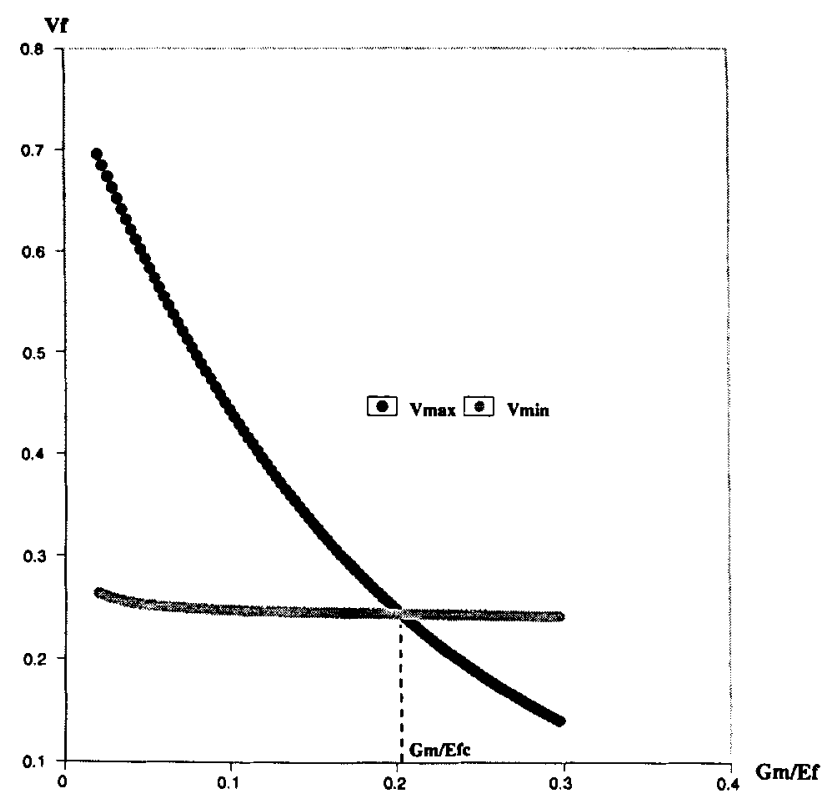

Fig. 9. Effects of the modulus ratio on volume fractions and the property compatibility boundary defined.

greater $\alpha$ value) of fiber orientation will lower the fiber orientation efficiency factor.

Figure 8 on the other hand indicates the effect of fiber orientation range $\alpha$ on the value of minimum fiber volume fraction $V_{\min }$. When $\alpha$ becomes larger, the value of $V_{\min }$ will increase, indicating that fiber reinforcing function is hampered due to fiber spreading so that more fibers are needed.

The curves in Figs. 9 and 10 can be used to test the property compatibility between the fibers and matrix. First of all, Fig. 9 shows the effects of the modulus ratio on the values of $V_{\max }$ (the same curve 


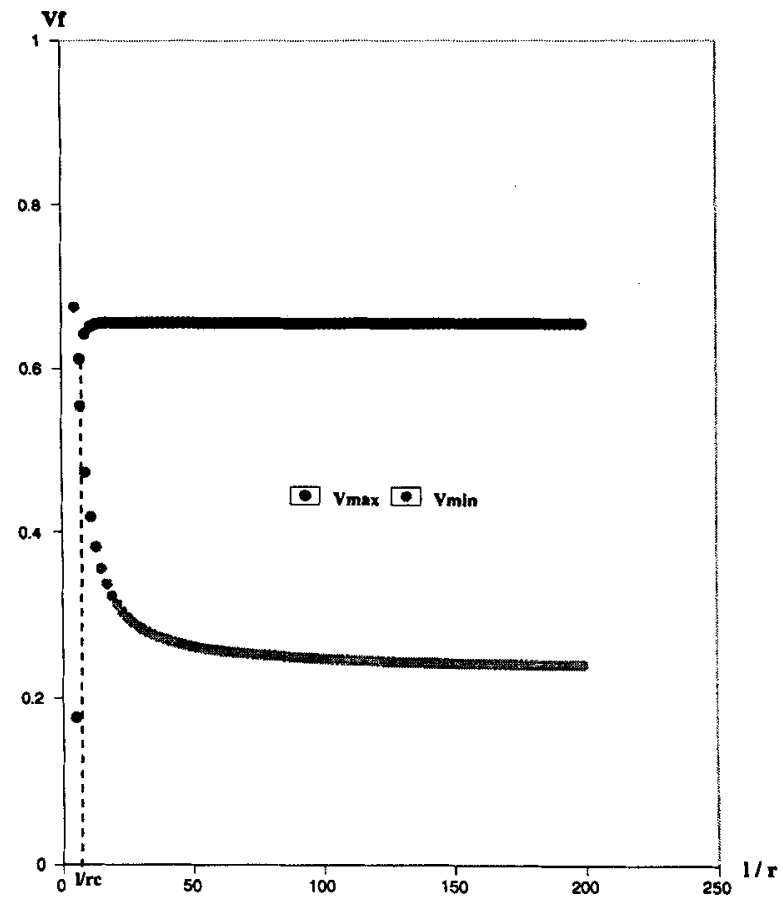

Fig. 10. Effects of the fiber aspect ratio on volume fractions and the property compatibility boundary defined.

as the short fiber case in Fig. 5) and $V_{m i n}$. Unlike $V_{\max }, V_{\min }$ decreases very slightly when $G_{m} / E_{f}$ is increasing. Based on $E q 40$, only those fiber and matrix types whose $G_{m} / E_{f}$ values are greater than the critical value $G_{m} / E_{f c}$ are compatible for being selected to form a properly functioning composite. In Fig. 10, first, the effects of $l / r$ on both volume fraction values $V_{\max }$ and $V_{\min }$ can be seen, showing different trends but both gradually approaching its own asymptote as $l / r$ increases. On the other hand. there is a critical value $l / r_{c}$ above which a feasible structure can be made.

\section{CONCLUSIONS}

The stress transfer between matrix and fibers in a composite is not only determined by the intrinsic properties of the fiber and matrix, but also affected by the geometric parameters of fiber arrangement within the matrix such as the spacing between fibers and the orientation of fibers. Consequently the shear strength of the interface between fibers and the ma- trix can be used as a criterion to study the fiber spacing effect in a composite.

For a composite made of given fiber and matrix materials, there is an optimal spacing between fibers at which the fiber tensile strength will be fully exploited. Moreover this optimal spacing is also the minimum allowable spacing between fibers below which the structure will start to disintegrate under loading before the fiber tensile failure. This minimum spacing then defines a maximum fiber volume fraction allowable for a composite. The maximum fiber volume fraction combined with the minimum fiber volume fraction studied previously can be used for composite design. Both volume fractions are found dependent on such parameters as fiber modulus $E_{f}$, fiber tensile strength $\sigma_{b f}$, fiber aspect ratio $l / r$ and fiber orientation range $\alpha$, the matrix properties as Poisson's ratio $\nu_{m}$, shear modulus $G_{m}$, and the bonding shear strength $\tau_{s}$ between fiber and matrix, as shown in this study. Consequently, these two values of fiber volume fraction $V_{\max }$ and $V_{\min }$ can be applied to define the boundaries in determining the property compatibility of various combinations of fiber and matrix types for a particular application so as to optimize the result of composite design.

\section{REFERENCES}

1. A. Bentur and S. Mindess, in Fiber Reinforced Cementi tious Composites, Elsevier Applied Science, New York (1990).

2. T. W. Chou and S. Nomura, Fiber Sci. and Techn. 14, $279(1980-81)$.

3. H. L. Cox, British J. of Appl. Phys., 3, 72 (1952).

4. P. T. Curtis, M. G. Bader, and J. E. Bailey, J. Mat. Sci., 13, 377 (1978)

5. C. F. Fan and S. Hsu, J. Polym. Sci.: Part B: Polym. Phys., 27, 2605 (1989)

6. C. F. Fan and S. Hsu, Macromolecules, 22, 1474 (1989).

7. A. Kelly and G. J. Davies, Metallurg. Reviews, 10, 1 (1965).

8. A. Kelly and N. H. McMillan, in Strong Solids, 3rd ed., Clarendon Press, Oxford (1986).

9. V. Laws, J. Phys. D; Appl. Phys., 4, 1737 (1971).

10. L. E. Malvern, in Introduction to the Mechanics of $A$ Continuous Medium, Prentice-Hall, Englewood Cliffs, N.J. (1969)

11. M. R. Piggott, in Load Bearing Fiber Composites, Pergamon Press, New York (1980).

12. B. W. Rosen, "Mechanics of Composite Strengthening," in Fiber Composite Materials, American Society for Metals (1965).

13. Y. Takao, T. W. Chou, and M. Taya, Transact. ASME, 49, 536 (1982).

14. N. Taya and T. W. Chou. J. Mat. Sci., 17, 2801 (1982). 